\title{
Venous Thromboembolism
}

\section{Deep Vein Thrombosis With Pulmonary Embolism, Deep Vein Thrombosis Alone, and Pulmonary Embolism Alone}

\author{
Masahito Sakuma, MD; Mashio Nakamura, MD*; Norikazu Yamada, MD*; \\ Satoshi Ota, MD*; Kunio Shirato, MD**; Takeshi Nakano, MD*; \\ Masaaki Ito, MD**; Takao Kobayashi, MD ${ }^{\dagger}$
}

\begin{abstract}
Background There are few data on the differences between deep vein thrombosis (DVT) with pulmonary embolism (PE) (Group A) and without PE (Group B), and no recent data on the incidence of PE and DVT in Japan. Methods and Results The symptoms and findings of the lower extremities and risks for venous thromboembolism were compared between Groups A and B, and the numbers of new patients with PE and those with DVT in 2006 were calculated. DVT was found equally in left and right legs in Group A, but more frequently in left legs than in right legs in Group B. Proximal thrombus was more frequent in Group A than in Group B, and the number of cases of symptoms resulting from DVT was less in Group A than in Group B. Proximal DVT, DVT in the right leg, no symptoms, and younger age were related to the presence of PE. The calculated number of new patients with PE per year was 7,864 (3,492 cases in 1996), and that with DVT per year was 14,674.

Conclusion DVT in patients with PE and those without PE differed in the site and symptoms. The calculated number of new patients with PE per year doubled in 1 decade in Japan. (Circ J 2009; 73: 305-309)
\end{abstract}

Key Words: Deep vein thrombosis; Incidence; Pulmonary embolism; Symptoms; Venous thromboembolism

$\mathbf{P}$ ulmonary embolism (PE) and deep vein thrombosis (DVT) are thought to be the same disease with different presentation, and both have been handled as venous thromboembolism (VTE). Most cases of PE originate from DVT, so VTE is an important concept. However, there are no data on whether DVT with PE and DVT without PE have the same characteristics.

We reported the incidence of PE in 1996, 2000, and 2004!-3 In 2004, 2 guidelines for VTE were published in Japan, ${ }^{4,5}$ generating increased interest in VTE.

The main purpose of this study was to clarify the different characteristics of DVT in cases with and without PE. The second purpose was to assess the recent incidence of PE and DVT in Japan.

\section{Methods}

The present study was approved by the Ethics Committee of Mie University. In July 2006, we sent questionnaires to the clinical departments (all departments of internal medicine, all departments of surgery, pediatrics, obstetrics and gynecology, orthopedics, otorhinolaryngology, ophthalmology, dermatology, and urology) of university schools of medi-

(Received April 16, 2008; revised manuscript received August 21, 2008; accepted September 11, 2008; released online December 18, 2008)

Division of Internal Medicine, Onagawa Municipal Hospital, Onagawa, *Department of Cardiology, Mie University Graduate School of Medicine, Tsu, **Division of Internal Medicine, Saito Hospital, Ishinomaki and ${ }^{\dagger}$ Hamamatsu Medical Center, Hamamatsu, Japan

Mailing address: Masahito Sakuma, MD, Division of Internal Medicine, Onagawa Municipal Hospital, 51-6 Horikiriyama, Washinokamihama, Onagawa 986-2243, Japan

All rights are reserved to the Japanese Circulation Society. For permissions, please e-mail: cj@j-circ.or.jp cine or medical colleges and to hospitals with more than 100 beds in Japan. Based on the responses to the questionnaires, we assessed prospectively the number of new patients with PE from August 1, 2006 to September 30, 2006. The number of patients with PE (or DVT) per year was calculated as: the number of patients with PE (or DVT) per year = the number of patients with PE (or DVT) per 2 months $\times 6 /$ the response rate! -3

PE was definitely diagnosed by (1) enhanced computed tomography, (2) pulmonary angiography, (3) pulmonary perfusion scintigraphy and/or pulmonary ventilation scintigraphy, (4) magnetic resonance imaging, or (5) autopsy. DVT was definitely diagnosed by (1) enhanced computed tomography, (2) venous ultrasonography, (3) contrast venography, (4) magnetic resonance venography, or (5) radioisotope venography. Major surgery was defined as abdominal surgery and/or surgery of more than 45 min duration within the previous 3 months ${ }^{6-8}$ Immobilization was defined as strict bed rest for more than 3 continuous days within the previous 3 months?

We divided cases of VTE into 3 groups: DVT with PE, DVT alone, and PE alone.

\section{Statistical Analysis}

Analyses were performed using SPSS 15.0 (SPSS Inc, Chicago, IL, USA). All continuous variables were analyzed by Mann-Whitney test, and expressed as mean \pm standard deviation. Non-ordinal categorical data were analyzed using the chi-square test. Multiple comparisons were performed using Bonferroni's modification. Potential risk factors for VTE were assessed using multiple logistic regression and the results were presented as estimated odds ratio (OR) with the corresponding 95\% confidence intervals (CI). All significant tests were 2 -tailed. 
Table 1 Patients' Backgrounds

\begin{tabular}{lccc}
\hline \hline & DVT with PE & DVT alone & PE alone \\
\hline With patient profile $(n)$ & 210 & 420 & 140 \\
Gender $(M / F)$ & $87 / 123$ & $140 / 280$ & $44 / 96$ \\
Age $($ years $)$ & $63.9 \pm 15.5$ & $66.3 \pm 15.9$ & $67.6 \pm 15.0$ \\
BMI $\left(\mathrm{kg} / \mathrm{m}^{2}\right)$ & $23.7 \pm 3.8^{a}$ & $23.3 \pm 4.2^{b}$ & $23.2 \pm 3.8^{c}$ \\
\hline
\end{tabular}

$a_{n}=199, b_{n}=392,{ }^{c} n=129$.

$D V T$, deep vein thrombosis; PE, pulmonary embolism; BMI, body mass index.

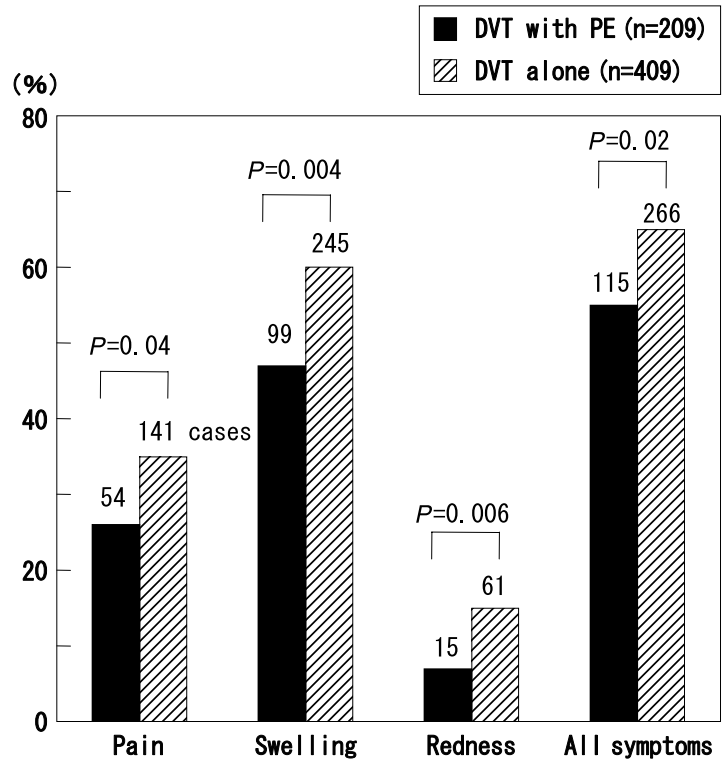

Fig 1. Symptoms of deep vein thrombosis (DVT). The number of cases is shown on each bar. PE, pulmonary embolism.

\section{Results}

Incidence of VTE

A total of 6,122 questionnaires were sent; 17 institutes were excluded from our analysis because they had closed or merged. We received 1,635 valid replies, giving a response rate of $26.8 \%(1,635 / 6,105)$. The number of patients newly diagnosed with PE was 351 during the 2 months of the present period, and that with DVT was 655 . The estimated number of new patients with PE per year was 7,864 (95\% CI: $6,572-9,155)$ and the incidence of PE was 61.9 (95\% CI: 51.7-72.1) patients per 1,000,000 people per year in Japan. The estimated number of new patients with DVT per year was 14,674 (95\% CI: 12,466-16,883) and the incidence of DVT was 115.5 (95\% CI: 98.2-132.9) patients per 1,000,000 people per year in Japan.

Characteristics of DVT in Patients With and Without PE

Available cases with a detailed profile were 210 with both DVT and PE, 420 with DVT alone, and 140 with PE alone (Table 1). Symptoms resulting from DVT were more frequent in patients without PE, compared with those with PE (Fig 1). DVT was equally found in the left and right legs
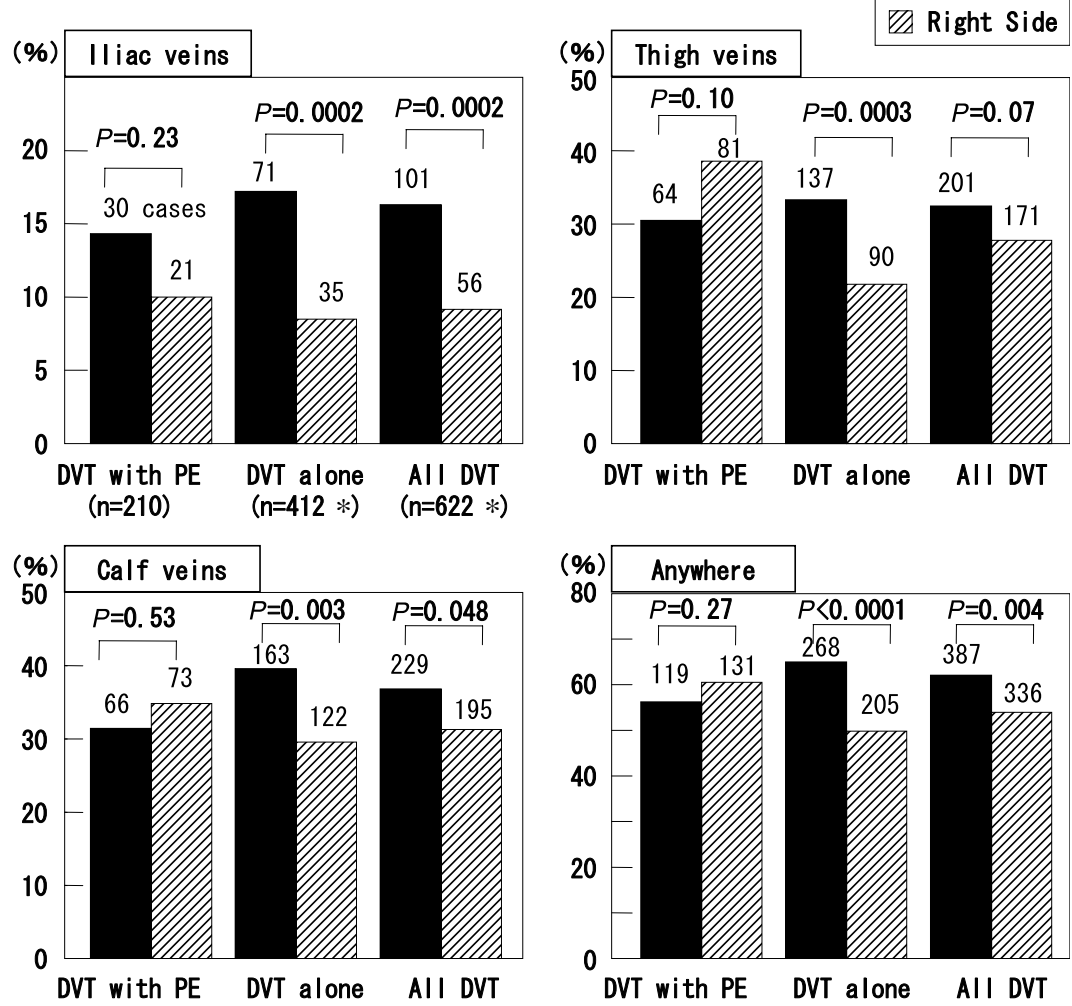

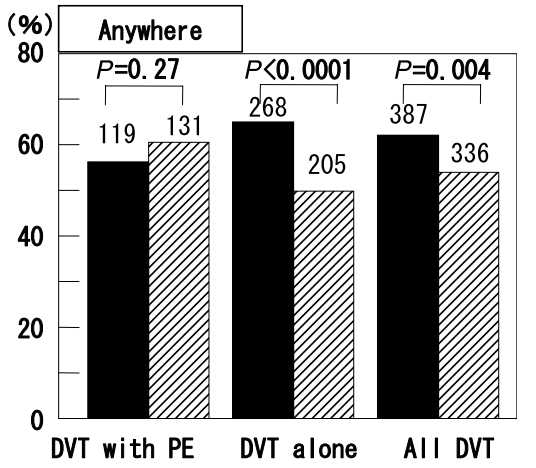

Fig 2. Location of deep vein thrombosis (DVT). The number of cases is shown on each bar. *Seven cases with DVT only in the upper extremities and one without data on DVT site were excluded. PE, pulmonary embolism. 
Table 2 Diagnostic Techniques for DVT

\begin{tabular}{lccc}
\hline \hline & $\begin{array}{c}\text { DVT with PE } \\
(n=210)\end{array}$ & $\begin{array}{c}\text { DVT alone } \\
(n=413 *)\end{array}$ & P value \\
\hline Venous ultrasonography & $132(63 \%)$ & $303(73 \%)$ & 0.008 \\
CT & $143(68 \%)$ & $180(44 \%)$ & $<0.0001$ \\
MR ventrast venography & $18(9 \%)$ & $50(12 \%)$ & 0.22 \\
RI venography & $7(3 \%)$ & $22(5 \%)$ & 0.32 \\
\hline
\end{tabular}

$C T$, computed tomography; MR, magnetic resonance; RI, radioisotope. Other abbreviations see in Table 1.

*Seven cases with DVT only in the upper extremities were excluded.

Table 3 Risk Factors for Venous Thromboembolism

\begin{tabular}{lcccc}
\hline \hline & $\begin{array}{c}\text { DVT with PE } \\
(n=210)\end{array}$ & $\begin{array}{c}\text { DVT alone } \\
(n=420)\end{array}$ & $\begin{array}{c}\text { PE alone } \\
(n=140)\end{array}$ & P value \\
\hline Prolonged immobilization & $57(27 \%)$ & $101(24 \%)$ & $30(21 \%)$ & 0.46 \\
Recent major surgery & $54(26 \%)$ & $121(29 \%)$ & $40(29 \%)$ & 0.70 \\
Cancer & $48(23 \%)$ & $81(19 \%)$ & $23(16 \%)$ & 0.32 \\
Recent major trauma and/or fracture & $22(11 \%)$ & $47(11 \%)$ & $15(11 \%)$ & 0.96 \\
Central venous catheter & $7(3 \%)$ & $34(8 \%)$ & $9(6 \%)$ & 0.06 \\
Pregnancy or postpartum & $4(2 \%)$ & $14(3 \%)$ & $2(1 \%)$ & 0.34 \\
Heart failure* & $5(2 \%)$ & $22(5 \%)$ & $14(10 \%)$ & 0.009 \\
Respiratory failure & $5(2 \%)$ & $14(3 \%)$ & $8(6 \%)$ & 0.37 \\
Cerebrovascular disease & $10(5 \%)$ & $28(7 \%)$ & $8(6 \%)$ & 0.62 \\
Connective tissue disease and/or steroid use & $5(2 \%)$ & $11(3 \%)$ & $5(4 \%)$ & 0.79 \\
Benign, large abdominal tumor & $2(1 \%)$ & $9(2 \%)$ & $1(1 \%)$ & 0.33 \\
No potential risk factors & $42(20 \%)$ & $66(16 \%)$ & $28(20 \%)$ & 0.30 \\
\hline
\end{tabular}

$* P=0.10$ between $D V T$ with $P E$ and $D V T$ alone, $P=0.003$ between DVT with $P E$ and $P E$ alone. $P=0.07$ between DVT alone and $P E$ alone. Abbreviations see in Table 1.

Table 4 Multivariate Logistic Analysis of Relation to Presence of PE in Patients with DVT

\begin{tabular}{lcc}
\hline \hline & OR $(95 \%$ CI $)$ & $P$ value \\
\hline Age (10-year increments) & $0.87(0.77-0.99)$ & 0.03 \\
Male & $1.12(0.76-1.66)$ & 0.57 \\
No symptoms of DVT & $2.05(1.39-3.02)$ & 0.0003 \\
Right DVT & $1.98(1.22-3.19)$ & 0.005 \\
Left DVT & $0.99(0.61-1.60)$ & 0.97 \\
Proximal DVTa & $1.79(1.18-2.71)$ & 0.006 \\
BMI & $1.03(0.99-1.08)$ & 0.16 \\
Prolonged immobilization & $1.2(0.78-1.86)$ & 0.41 \\
Recent major surgery & $0.83(0.54-1.28)$ & 0.40 \\
Cancer & $1.08(0.68-1.70)$ & 0.75 \\
Recent major trauma and/or fracture & $0.85(0.46-1.54)$ & 0.58 \\
Central venous catheter & $0.44(0.19-1.00)$ & 0.05 \\
Pregnancy or postpartum & $0.37(0.10-1.32)$ & 0.12 \\
Heart failure & $0.59(0.20-1.68)$ & 0.32 \\
Respiratory failure & $0.58(0.18-1.92)$ & 0.37 \\
Cerebrovascular disease & $0.66(0.28-1.55)$ & 0.34 \\
Connective tissue disease and/or steroid use & $1.48(0.54-4.02)$ & 0.45 \\
Benign, large abdominal tumor & - & 1.00 \\
\hline
\end{tabular}

Including IVC, iliac vein, and thigh veins.

$O R$, odds ratio; CI, confidence interval; IVC, inferior vena cava. Other abbreviations see in Table 1.

of patients with $\mathrm{PE}$, but more frequently in the left than in the right leg of patients without PE (Fig 2). Proximal thrombus from the inferior vena cava to the popliteal vein was more frequent in patients with $\mathrm{PE}$ than in patients without $\mathrm{PE}(68 \%$ [142/210] vs 58\% [240/412]; $\mathrm{P}=0.02)$.

\section{Relationship Between Symptoms of DVT and Age}

Leg swelling (presence, $64.7 \pm 16.0$ years; absence, $66.5 \pm$ $15.7 ; \mathrm{P}=0.10$ ) and redness (presence, $63.0 \pm 15.7$ years; absence, 65.9 $\pm 15.9 ; \mathrm{P}=0.09$ ) were found regardless of age in patients with DVT. Younger patients complained more about

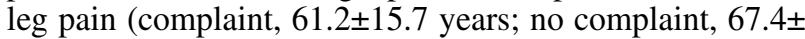

15.6; $\mathrm{P}<0.0001$ ). All findings for DVT (objective or subjective) were greater in younger patients (presence, $64.4 \pm 15.8$ years; absence, $67.4 \pm 15.9 ; \mathrm{P}=0.007)$.

\section{Diagnostic Techniques for DVT (Table 2)}

Venous ultrasonography was used more frequently and CT less frequently in patients without PE than in patients with PE. Contrast venography was used in only approximately $10 \%$ of patients.

Risk Factors for VTE and Relationship to Presence of PE There were no differences in the risk factors, except heart 
Table 5 Management of Venous Thromboembolism

\begin{tabular}{lcccccc}
\hline \hline & \multirow{2}{*}{$\begin{array}{c}\text { DVT with PE } \\
(n=210)\end{array}$} & $\begin{array}{c}{ }^{2} \text { DVT alone } \\
(n=420)\end{array}$ & $\begin{array}{c}{ }^{3} \text { PE alone } \\
(n=140)\end{array}$ & & \multicolumn{3}{c}{$P$ value* } \\
\cline { 6 - 7 } & $175(83 \%)$ & $243(58 \%)$ & $106(76 \%)$ & $<0.0001$ & 0.30 & 0.0005 \\
Heparin & $162(77 \%)$ & $282(67 \%)$ & $86(61 \%)$ & 0.03 & 0.006 & 0.66 \\
Warfarin & & & & & & \\
Anticoagulation & $136(65 \%)$ & $173(41 \%)$ & $69(49 \%)$ & $<0.0001$ & 0.02 & 0.30 \\
$\quad$ Heparin $\rightarrow$ warfarin & $39(19 \%)$ & $70(17 \%)$ & $37(26 \%)$ & 1.00 & 0.26 & 0.04 \\
$\quad$ Heparin alone & $26(12 \%)$ & $109(26 \%)$ & $17(12 \%)$ & $<0.0001$ & 1.00 & 0.003 \\
$\quad$ Warfarin alone & $58(28 \%)$ & $55(13 \%)$ & $38(27 \%)$ & $<0.0001$ & 1.00 & 0.006 \\
Thrombolysis & $110(52 \%)$ & $93(22 \%)$ & $22(16 \%)$ & $<0.0001$ & $<0.0001$ & 0.35 \\
IVC filter & & & & &
\end{tabular}

*All P-values by chi-square analysis among 3 groups $\left({ }^{1},{ }^{2}\right.$ and $\left.{ }^{3}\right)$ were less than 0.05 . Multiple comparisons were performed using Bonferroni's modification.

Abbreviations see in Tables 1, 4 .

failure, among the 3 groups (patients with DVT and PE, those with DVT alone, and those with PE alone) (Table 3). Patients with DVT and PE were younger than those with DVT alone $(63.9 \pm 15.5$ years vs $66.3 \pm 15.9 ; \mathrm{P}=0.04)$. $\mathrm{PE}$ was found in $30.5 \%$ of females with DVT and in $38.3 \%$ of males with DVT $(\mathrm{P}=0.053)$. Proximal DVT, DVT in the right leg, no symptoms, and younger age were independently related to the presence of PE in patients with DVT (Table 4).

\section{Management of VTE (Table 5)}

Heparin and thrombolysis were used less frequently in patients with DVT alone. Implantation of an inferior vena cava filter and chronic use of warfarin were more frequent in patients with DVT and PE. When limited to cases of DVT, inferior vena cava filters were used more often in cases of proximal DVT (OR, 3.51; 95\% CI, 2.33-5.27; P<0.0001) and $\mathrm{PE}(\mathrm{OR}, 3.71$; 95\% CI, 2.56-5.37; $\mathrm{P}<0.0001)$. Antiplatelet agents were administered in 8 patients (4\%) with DVT and PE (aspirin in 8, ticlopidine in 2; 2 cases used both antiplatelet agents), 44 with DVT alone (aspirin in 36, ticlopidine in 6, cilostazol in 1, sarpogrelate in 1), and 9 (6\%) with PE alone (aspirin in 8, ticlopidine in 1, beraprost in 2; 2 cases used 2 antiplatelet agents).

For DVT, catheter therapy was performed in 9 patients with DVT and PE, and in 8 patients with DVT alone. Surgery was performed in 3 patients with DVT and PE, and in 1 patient with DVT alone. On the other hand, for PE, catheter therapy was performed in 13 patients with DVT and PE, and in 7 patients with PE alone. Surgery was performed in 4 patients with DVT and PE, and in 4 patients with PE alone.

\section{Discussion}

\section{Characteristics of DVT With and Without PE}

DVT in patients with PE and those without PE differed in the site and symptoms. In particular, DVT was equally found in the left and right legs of patients with PE, but more frequently in the left than in the right leg in those without PE. Moreover, cases of symptoms resulting from DVT were less frequent in the presence of PE than in the absence of PE.

Ileofemoral DVT tends to occur in the left leg, ${ }^{-12}$ whereas femoropopliteal DVT occurs equally in the right and left legs, and most are contiguous to calf thrombosis?-12 Those previous reports and the present results suggest that DVT without PE is related to ileofemoral DVT, and that DVT with PE is related to femoropopliteal DVT.

DVT is more common on the left side, ${ }^{13}$ as observed in all of the present cases of DVT. In the present study, DVT with PE had no statistical difference in the rate of potential risk factors compared with DVT without PE.

Free-floating venous thrombi have a close relationship with PE compared with occlusive (no free-floating) thrombi ${ }^{14}$ and the previous reports suggest that free-floating venous thrombi cause less symptoms from DVT than occlusive DVTs ${ }^{14,15}$ On the other hand, most cases of symptomatic DVT have extensive occlusive proximal thrombi9,16 The development of symptoms of DVT is thought to depend on the extent of thrombosis, the adequacy of collateral vessels, and the severity of associated vascular occlusion and inflammation. ${ }^{17}$ Leg edema is much more likely in contiguous thrombosis rather than with an isolated thrombus. ${ }^{8}$ DVT with PE has fewer symptoms, as shown in the present study, and resembles free-floating DVT.

\section{Relationship to Presence of PE in Patients With DVT}

Proximal DVT, DVT in the right leg and no symptoms of DVT were identified as independent of the presence of PE. Proximal DVT is often associated with acute PE ${ }^{19-23}$ Embolic risk is low in calf-only DVT, but elevated in calf DVT with proximal (thigh) involvement 19 DVT in the right iliac vein is easily torn off and PE easily occurs because the right iliac vein is not compressed, unlike the left iliac vein. Most cases of DVT with no symptoms do not receive treatment and in such cases the DVT is found after PE occurs, which suggests that DVT showing few symptoms is a potential risk for PE. One of the candidate DVT is free-floating thrombi, but further study is needed to clarify this. Older patients with DVT have fewer symptoms and less incidence of PE; they may have fewer symptoms of PE and not be diagnosed as such, even if they have PE, but the real reason is unknown.

In the present study, the incidence of DVT was the same for the right and left legs in patients with PE, but multivariate logistic analysis revealed that DVT in the right leg was a risk for PE, because the left leg was prominent in all patients with DVT.

\section{Diagnostic Techniques for DVT}

Venous ultrasonography was used more frequently and CT less frequently in patients without PE than in patients with PE. Venous ultrasonography is noninvasive and convenient, and many diagnostic strategies for DVT use this method5,24 CT has been used more recently for the diagnosis of $\mathrm{PE}$ in recent years, 25 as its sensitivity for $\mathrm{PE}$ is not inferior to ventilation-perfusion lung scanning $26 \mathrm{CT}$ has the merit that DVT is diagnosed at the same time, so many doctors in Japan may choose venous ultrasonography as the initial diagnostic method in patients suspected of having DVT, and CT in patients suspected of having PE. 


\section{Management of VTE}

Heparin and thrombolysis were used less frequently in patients with DVT alone. Chronic use of warfarin was more frequent in patients with DVT and PE. Moreover, warfarin was used first more frequently without heparin in cases of DVT alone.

Implantation of an inferior vena cava filter was more frequently performed in patients with DVT and PE. When limited to cases of DVT, inferior vena cava filters were more frequently used in proximal DVT with PE. Recurrence of PE in a patient with PE would increase mortality, so inferior vena cava filters are used to prevent recurrent PE in patients with both DVT and PE.

\section{Incidence of VTE}

The calculated number of new patients with PE per year was 3,492 cases in $1996^{1}$ and 7,864 in 2006 in the present study. The calculated number of new patients with PE per year increased 2.25-fold in 1 decade in Japan. These results are similar to the prevalence of PE estimated by the Ministry of Health, Labour and Welfare in Japan (3,000 patients in 1996 and 7,000 in 2005)?27,28 The vital statistics were 1,410 deaths from PE in 1996, and 1,900 deaths in 200629,30 Annual deaths from PE increased 1.35-fold in 1 decade, which was lower than the increment of diagnostic patients during the same period.

The calculated number of new patients with DVT per year was 14,674 in 2006 , which is similar to the prevalence reported in $2005\left(16,000\right.$ cases $\left.^{28}\right)$.

\section{Study Limitations}

One limitation of the present study is the low response rate. Response rates for questionnaires regarding less common diseases are low in general. The response rate in studies on the incidence of PE performed by us was $40.7 \%$ in $1996,30.6 \%$ in $2000,29.8 \%$ in 2004 , and $26.8 \%$ in the present study.

Our results may be affected by the timing of the diagnosis and examination of VTE. Moreover, symptoms of PE may mask symptoms of DVT, despite this being a prospective study. Therefore, additional examinations are necessary to confirm the present results.

\section{Conclusion}

DVT in patients with and without PE differs in its site and symptoms. The calculated number of new patients with PE per year doubled over 1 decade in Japan.

\section{Acknowledgment}

This study was partly supported by a grant from the Japanese Ministry of Health, Labor and Welfare for Blood Coagulation Abnormalities.

\section{References}

1. Kumasaka N, Sakuma M, Shirato K. Incidence of pulmonary thromboembolism in Japan. Jpn Circ J 1999; 63: 439-441.

2. Kitamukai O, Sakuma M, Takahashi T, Kagaya Y, Watanabe J, Shirato K. Incidence and characteristics of pulmonary thromboembolism in Japan 2000. Intern Med 2003; 42: 1090-1094.

3. Sugimura K, Sakuma M, Shirato K. Potential risk factors and incidence of pulmonary thromboembolism in Japan: Results from an overview of mailed questionnaires and matched case-control study. Circ J 2006; 70: $542-547$.

4. Editorial Committee on Japanese Guideline for Prevention of Venous Thromboembolism. Japanese Guideline for Prevention of Venous Thromboembolism. Medical Front International Ltd, Tokyo, 2004 (in Japanese).
5. The Japanese Circulation Society. Guidelines for the diagnosis, treatment and prevention of pulmonary thromboembolism and deep vein thrombosis (JCS 2004). Circ J 2004; 68(Suppl): IV-1079-IV-1134 (in Japanese).

6. Nicolaides A, Breddin H, Fareed J, Goldhaber S, Haas S, Hull R, et al. Prevention of venous thromboembolism: International Consensus Statement: Guidelines compiled in accordance with the scientific evidence. Int Angiol 2001; 20: 1-37.

7. Heit JA, Silverstein MD, Mohr DN, Petterson TM, O'Fallon WM, Melton LJ 3rd. Predictors of survival after deep vein thrombosis and pulmonary embolism. Arch Intern Med 1999; 159: 445-453.

8. Quinn DA, Thompson BT, Terrin ML, Thrall JH, Athanasoulis CA, McKusick KA, et al. A prospective investigation of pulmonary embolism in women and men. JAMA 1992; 268: 1689-1696.

9. Cogo A, Lensing AW, Prandoni P, Hirsh J. Distribution of thrombosis in patients with symptomatic deep vein thrombosis: Implications for simplifying the diagnostic process with compression ultrasound. Arch Intern Med 1993; 153: $2777-2780$.

10. Nylander G, Olivercrona H. The phlebographic pattern of acute leg thrombosis within a defined urban population. Acta Chir Scand 1976; 142: $505-511$

11. Stamatakis JD, Kakkar VV, Lawrence D, Bentley PG. The origin of thrombi in the deep veins of the lower limb: A venographic study. $\mathrm{Br}$ J Surg 1987; 65: 449-451.

12. Nicolaides AN, Kakkar VV, Field ES, Renney JT. The origin of deep vein thrombosis: A venographic study. Br J Radiol 1971; 44: $653-$ 663.

13. Ouriel K, Green RM, Greemberg RK, Clair DG. The anatomy of deep venous thrombosis of the lower extremity. J Vasc Surg 2000; 31: $895-900$.

14. Norris CS, Greenfield LJ, Herrmann JB. Free-floating ileofemoral thrombus: A risk of pulmonary embolism. Arch Surg 1985; 120: 806-808.

15. Baldridge ED, Martin MA, Welling RE. Clinical significance of freefloating venous thrombi. J Vasc Surg 1990; 11: 62-67.

16. Markel A, Manzo RA, Bergelin RO, Strandress ED Jr. Pattern and distribution of thrombi in acute venous thrombosis. Arch Surg 1992; 127: $305-309$.

17. Kearon C. Natural history of venous thromboembolism. Circulation 2003; 107(Suppl): I-22-I-30.

18. Hill SL, Holtzman GI, Martin D, Evans P, Toler W, Goad K. The origin of lower extremity deep vein thrombi in acute venous thrombosis. Am J Surg 1997; 173: 485-490.

19. Moser KM, LeMoine JR. Is embolic risk conditioned by location of deep venous thrombosis? Ann Intern Med 1981; 94: 439-444.

20. Ferris EJ. Peripheral deep venous thrombosis and pulmonary thromboembolism: Correlative diagnostic evaluation. Int Angiol 1983; 2: $85-98$.

21. Dorfman GS, Cronan JJ, Tupper TB, Messersmith RN, Denny DF, Lee CH. Occult pulmonary embolism: A common occurrence in deep vein thrombosis. Am J Roentgenol 1987; 148: 263-266.

22. Hiusman MV, Buller HR, ten Cate JW, van Roven EA, Vreeken J, Kersten MJ, et al. Unexpected high prevalence of silent pulmonary embolism in patients with deep venous thrombosis. Chest 1989; 95: $498-502$.

23. Monreal M, Ruiz J, Olazabal A, Arias A, Roca J. Deep venous thrombosis and the risk of pulmonary embolism: A systematic study. Chest 1992; 102: 677-681.

24. Zieler BK. Ultrasonography and diagnosis of venous thromboembolism. Circulation 2004; 109(Suppl): I-9-I-14.

25. Sakuma M, Okada O, Nakamura M, Nakanishi N, Miyahara Y, Yamada N, et al. Recent developments in diagnostic imaging techniques and management for acute pulmonary embolism: Multicenter registry by the Japanese Society of Pulmonary Embolism Research. Intern Med 2003; 42: 470-476.

26. Anderson DR, Kahn SR, Rodger MA, Kovacs MJ, Morris T, Hirsch $\mathrm{A}$, et al. Computed tomographic pulmonary angiography vs ventilation-perfusion lung scanning in patients with suspected pulmonary embolism. JAMA 2007; 298: 2743-2753.

27. Statistics and Information Department, Minister's Secretariat, Ministry of Health, Labour and Welfare. Patient survey 1996. Tokyo, 1998.

28. Statistics and Information Department, Minister's Secretariat, Ministry of Health, Labour and Welfare. Patient survey 2005. Tokyo, 2007.

29. Statistics and Information Department, Minister's Secretariat, Ministry of Health, Labor and Welfare. Vital statistics of Japan 1996. Tokyo, 2008.

30. Statistics and Information Department, Minister's Secretariat, Ministry of Health, Labor and Welfare. Vital statistics of Japan 2006. Tokyo, 2008. 